\title{
Experimental and Computational Analysis of Shock Structure in a Supersonic Contoured Nozzle
}

\author{
Jijo Unnikrishnan \\ Staff Instructor \\ Faculty of Air Engg \\ NIAT, Cochin, Kerala
}

\author{
Vyshnav Vasantakumar \\ PG Scholar \\ Faculty of Air Engg \\ NIAT, Cochin, Kerala
}

\author{
Harshal Patil \\ PG Scholar \\ Faculty of Air Engg \\ NIAT, Cochin, Kerala
}

\begin{abstract}
Flow separation in a supersonic contoured nozzle whilst operating under over expanded regime is an inevitable fluid dynamic phenomenon. The flow field comprise formation of complex shock structures and its interaction with the viscous boundary layer. Profound number of researches on various types of contoured nozzle profiles have been undertaken to better understand the said phenomenon. The present study is focused on further understanding the fundamentals associated with formation of shocks and its structural transformation under varying NPR for a defined area ratio. Flow visualization utilizing Schlieren photography has enabled to capture the details of shock and its structure along with other phenomenon viz boundary layer separation, shock boundary layer interaction, aftershocks etc. The locations of shock captured experimentally have also been ascertained with computational generated data for various NPR and the results have been found quite comparable.
\end{abstract}

Keywords-Nozzle, supersonic, overexpansion, contour, lambda feet, area ratio, NPR, SBLI, FSS, RSS, Ansys-Fluent, oblique shock, reflected shock, Schlieren photography, flow visualization, boundary layer, pressure gradients, lambda feet, triple point, slipstream, shear layer, aftershock

\section{INTRODUCTION}

Flow separation in a supersonic nozzle is a basic fluid dynamic problem wherein the viscous flow adjusts to its ambient conditions through a non-isentropic, discontinuous shock jump [1-2]. Supersonic flow inside rocket nozzle during its course of operation in the over expanded region ie, exit pressure less than ambient pressure, adjusts itself to the ambient conditions by developing complex shock structure, by virtue of which, flow gets separated from the nozzle walls due to adverse pressure gradients.

A detailed investigation on separated flow in nozzles by Nasuti et al clearly describes the attributes of the shock structure [3]. The Mach disk of lambda shock changes shape as the NPR increases. For low NPR, it appears as normal shock whereas for moderate NPR and above, it becomes either convex or concave. This phenomenon is attributable to the presence of non-uniformities in the upstream flow. The gradients in the upstream flow (positive or negative, both radially and axially) largely influence the shape of the Mach disk. This non-uniformity in the upstream flow i.e., ahead of the shock can even generate a vortex in the core center flow such that the supersonic flow behind the shock bends towards the nozzle wall, creating reattachment. Asymmetry in the said reattachment phenomenon can create side loads on the nozzle walls $[3,4]$. Therefore, for a supersonic nozzle operating in the over expanded regime, its performance characteristics are largely defined by the shock structure. Classical, inviscid, onedimensional supersonic nozzle flow, explained in text books, define this structure as a simple normal shock with flow downstream of the shock attached to the nozzle walls, whereas in actual practice, it is a case of strong interaction of oblique shock waves with viscous boundary layer, thereby developing a complex shock structure.

The types of flow separation in rocket nozzles are categorized as Free Shock Separation (FSS), wherein the flow downstream of the shock structure is detached from the nozzle wall and never attaches back, and Restricted Shock Separation (RSS), in which flow downstream of the shock structure attaches back to the nozzle wall. The shock wave boundary layer interaction (SBLI) exhibits strong unsteadiness and unsymmetrical flow separation, leading to unsymmetrical circumferential pressure distribution on the nozzle walls, called side loads [4]. The existence of particular type of flow separation depends on the type of shock structure generated within the nozzle, which in turn is attributed to the type of profile and Nozzle Pressure Ratio (NPR); and it has been experimentally proven that the NPR corresponding to transition from RSS to FSS and its hysteresis creates asymmetric pressure distribution on the nozzle walls, leading to asymmetric side loads which can even damage the nozzle hardware [5].

Myriads of research have been undertaken to capture the shock structures in different nozzle profiles under different operating conditions and to ascertain the fundamental mechanism behind such structures. Of the prominent researches, Papamoschou et al has elucidated the fundamentals of shock structure in a supersonic planar nozzle using high quality Spark Schlieren photography [6]. The complex structure, often called a lambda shock, formed at moderate NPR, is a pure depiction of Mach reflection, comprising of an incident shock, a reflected shock and a Mach disk at the center. The point where incident and reflected shock meet is called the triple point, from where the slipstream or entropy layer is emanated. A shear layer originates from the separation point and extends downstream, detaching the boundary layer from the nozzle wall. As the NPR increases, the lambda feet grow bigger and becomes asymmetric, thus, making the separation shear layer highly unstable, which subsequently grows into large eddies in the flow downstream. 
The present study focusses on understanding the shock structure inside a contoured supersonic nozzle with area ratio $\left(\mathrm{A}_{\mathrm{e}} / \mathrm{A}_{\mathrm{t}}\right)=1.62$ and design Mach number, $\mathrm{M}=2.25$ under varying NPR. The shock structure has been investigated using Schlieren photography. The experiments were aimed at capturing phenomena viz lambda shock formation and its location, shock reflection and aftershocks. Computational analysis using commercial CFD package Fluent has also been undertaken to simulate the flow process and to compare the shock locations for various NPR.

\section{EXPERIMENTAL SETUP}

\section{A. Nozzle Design}

A supersonic contoured nozzle with profile, shown in fig. 1 , was designed and fabricated to analyze the shock structure. The 2-D nozzle profiles were wire cut and fabricated out of mild steel. The profile contours equations of various regions with length $\mathrm{x}$ (in $\mathrm{mm}$ ) as variable are shown in table 1 .

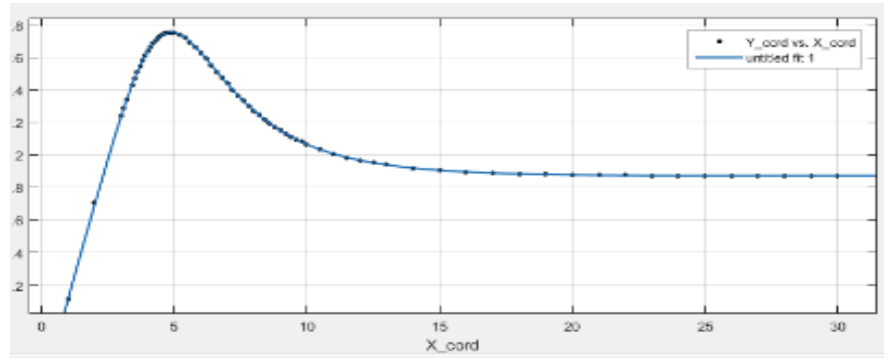

Fig.1. Nozzle Profile.

TABLE 1. PROFILE EQUATIONS

\begin{tabular}{|c|c|}
\hline $\begin{array}{c}\text { Nozzle } \\
\text { Regions }\end{array}$ & Profile \\
\hline Region I & $\mathrm{y}=0.7801 \mathrm{x}-0.05249 \mathrm{x}^{2}$ \\
& $\mathrm{x} \in[0,3.5]$ \\
\hline Region II & $\mathrm{y}=-2.361511+2.363743 \mathrm{x}-$ \\
& $0.3730563 \mathrm{x}^{2}+0.01785859 \mathrm{x}^{3}$ \\
& $\mathrm{x} \in[3.5,6]$ \\
\hline Region III & $\mathrm{y}=4.8081-0.6556 \mathrm{x}+0.04487 \mathrm{x}^{2}-0.001049 \mathrm{x}^{3}$ \\
& $\mathrm{x} \in[6,16]$ \\
& \\
\hline
\end{tabular}

\section{B. Experimental Arrangement}

The experiments were carried out on a blow down supersonic wind tunnel as shown in Fig. 2 at Naval Institute of Aeronautical Technology, Cochin. The flow inlet connected to an external reservoir, was charged by a HP compressor and the flow to the settling chamber was regulated by a precision operating valve. The settling chamber had pressure tapping provision for stagnation pressure $\left(\mathrm{P}_{0}\right)$ and was designed in such a way to provide uniform flow to the test section. The stagnation pressure $\mathrm{P}_{0}$ indicated by the pressure gauge was varied using the operating valve. The nozzle profiles were mounted on to the base plate using Allen screws. In order to make the test section transparent for Schlieren visualization, the base plates made of mild steel were provisioned with two slots for holding the glass window. Two extra clear glasses of $12 \mathrm{~mm}$ thickness were tight fitted in these slots. The nozzle base plate assembly was mounted onto the stagnation chamber using flange made of mild steel.

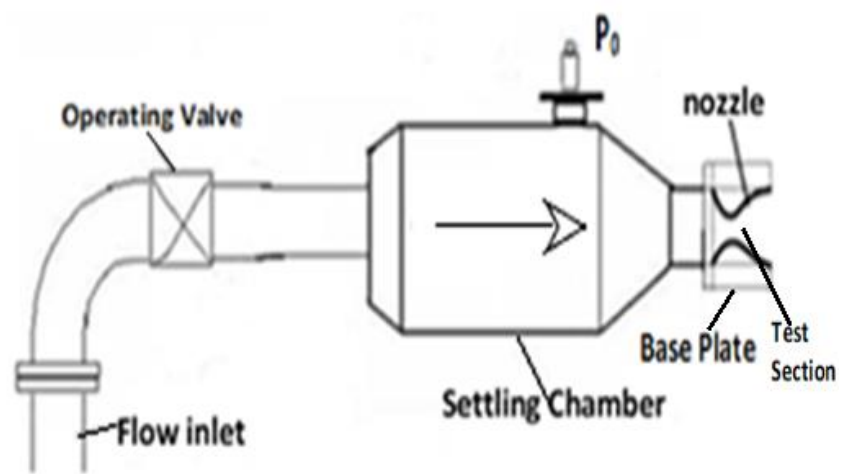

Fig.2. Schematic of blow down tunnel set up.

The nozzle flow field was visualized by means of Schlieren optical method wherein the change in refractive index due to density variation is used to map the change in flow field. This method measures the first derivative of density gradient unlike shadowgraph which measures the second gradient [7-9]. For the Schlieren visualization, a point light source, monochromatic halogen lamp of $150 \mathrm{~W}$, two concave mirrors of diameter $75 \mathrm{~mm}$, gimbal mounts, adjustable apertures, knife edge and a DSLR camera with frame rate of 60 frames per second were used. Intensity of light falling on the camera was controlled using a knife edge, placed at the focal length of the second mirror. Shock waves being regions of high density, beams of parallel light rays bend, as it passes through regions of density variation (due to change in refractive index). The refracted light rays thus deviate from the parallel path and are do not get blocked by the knife edge, forming a bright image of shock wave on the camera. The experimental arrangement is shown in fig 3.

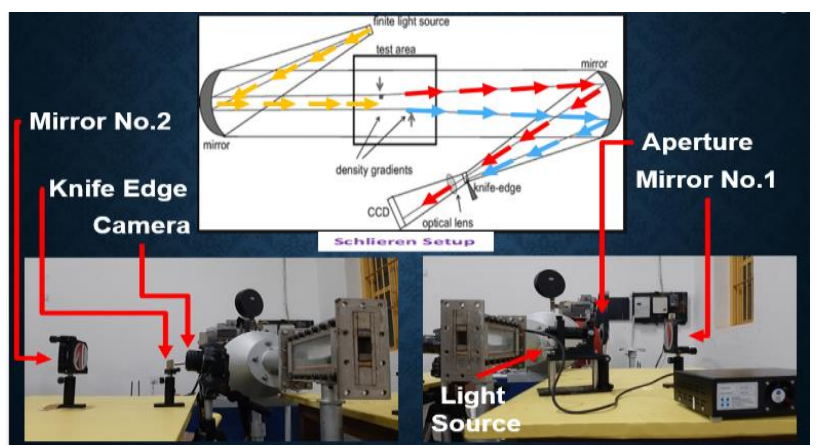

Fig 3. Experimental set up.

\section{EXPERIMENTAL ANALYSIS}

\section{A. Shock Visualisation}

The flow field inside the contoured nozzle is a function of NPR. Schlieren photographs of flow field with increasing NPR, captured using a vertical knife edge, are shown in sequence in fig 4(a) to 5(h). For NPR $<1.15$, the nozzle was not choked and the flow downstream was subsonic as indicated in fig 5(a). For NPR $=1.2$, weak normal shocks were observed downstream of throat as shown in fig 5(b). Above NPR corresponding to 1.3 , existence of normal shock was more prominent. With the increase in NPR $>1.4$, the shock developed 'lambda feet' as shown in fig 5 (e). The formation of lambda 
feet was asymmetrical as evident in fig 5(f) wherein the bottom wall attained lambda feet first as compared to the top wall. This phenomenon was inconsistent i.e, in the subsequent runs, the asymmetry flipped (top wall attained lambda feet first as compared to bottom wall). For NPR> 1.5, the lambda feet became stronger and the Mach disk attained convex shape. For NPR $>1.6$, strong boundary layer separation was observed with flow separation initiated from the first leg of lambda feet i.e., from the incipient incident shock. The Mach disk became more convex with the increase in the slope of incident shocks once NPR reached 1.7 as shown on fig $4(\mathrm{~h})$.
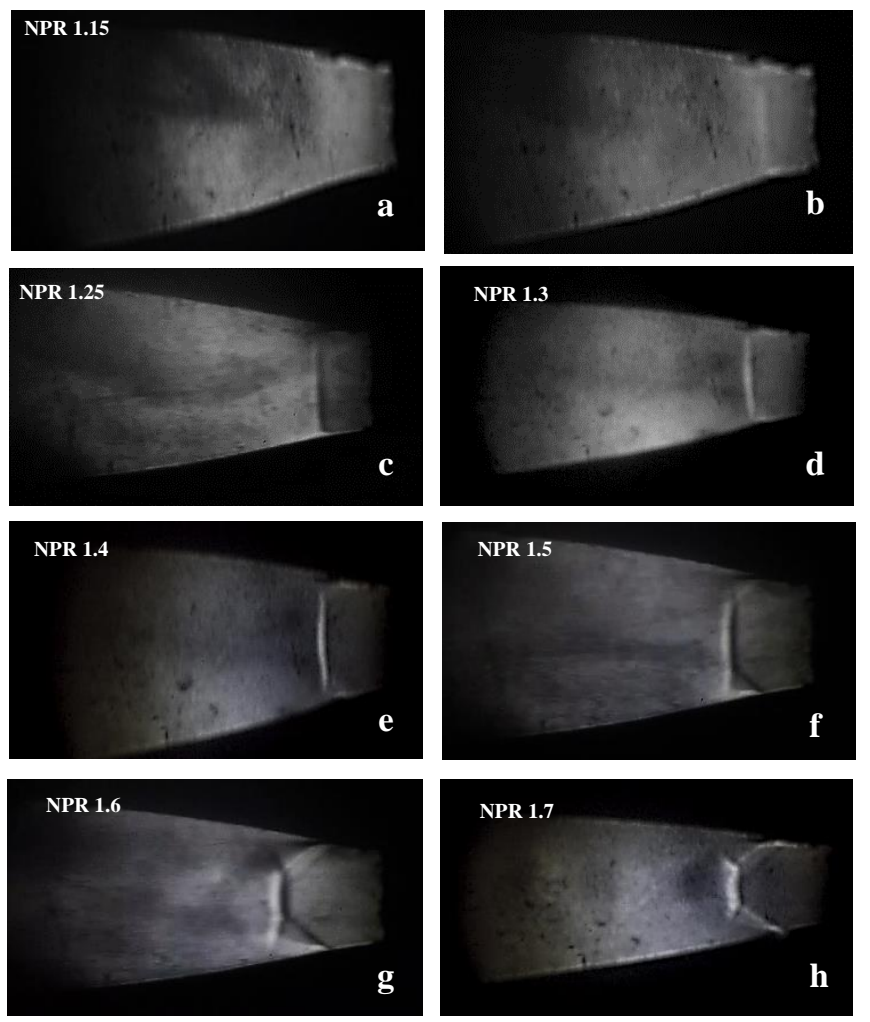

Fig.4 Shock visualisation.

\section{B. Shock Structure}

At NPR above 1.5, the shock attained a complex structure called lambda shock as shown in fig 5. The lambda structure comprised of the following: -

$\begin{array}{lll}\mathrm{a} & - & \text { Mach disk } \\ \mathrm{b} & - & \text { incident shock } \\ \mathrm{c} & - & \text { reflected shock } \\ \mathrm{t} & - & \text { triple point }\end{array}$

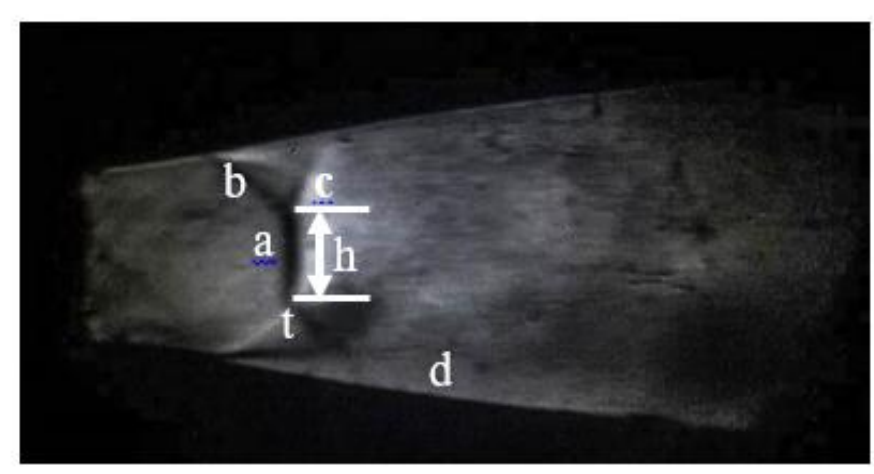

Fig. 5 Shock Structure

The supersonic viscous flow inside contoured nozzle, whilst operating under over expanded conditions, developed a strong oblique shock called incident shock (b), towards attaining equilibrium with the ambient conditions. Since the slope of the incident shock increased towards axis of symmetry, thereby increasing its strength, the only possible reflection was Mach reflection. Thus, the incident shock was observed to reflect as a Mach reflection with (c) as the reflected shock and (t) being the triple point where both the incident and reflected shocks were merged. A convex shaped Mach disk (a) was formed with height (h) as the height of the Mach stem i.e, the normal part of the Mach disk.

The first foot of the lambda shock i.e., the starting point of the incident shock (separation point), induced a strong adverse pressure gradient, which was strong enough to cause boundary layer separation as shown in (d). A shear layer originated from the separation point whose thickness increased with the increase in NPR. This shear layer caused the flow to remain detached downstream and continue as free jet. A slipstream known as entropy layer emanated from the triple point and continued downstream. A better comprehension of both shear layer and slipstream can be understood from the schematic fig 6 .

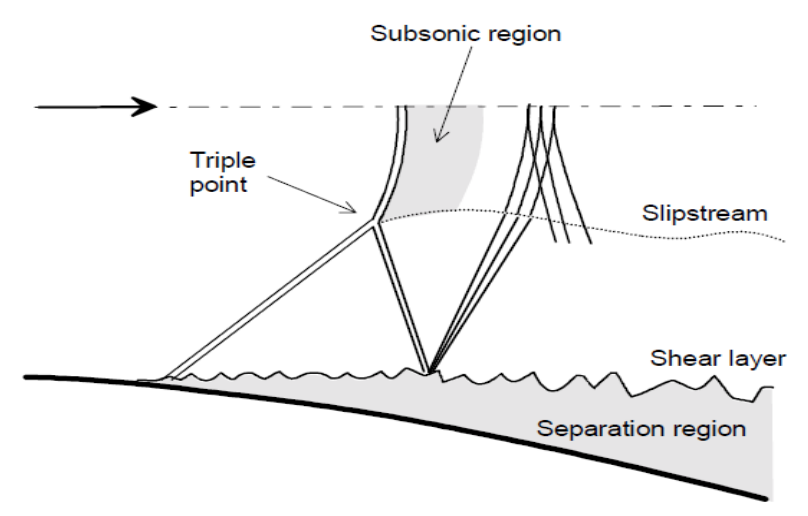

Fig. 6. Separation schematic [6]

At low NPR ie, $1.2 \leq \mathrm{NPR} \leq 1.5$ the separation shock formed was a weak normal shock and for NPR $\geq 1.6$, the Mach disk attained convex shape, wherein, in the ideal case, it would have been a straight line. This phenomenon was attributed to the flow non-uniformities. Fully stabilised uniform flow cannot be ensured by the stagnation chamber and the upstream flow is prone to have non-uniformities, induced by pipe friction, 
viscosity etc. Thus, the shock structure is actually a case of non-uniform Mach reflection from axis of symmetry.

\section{Non-Uniform Mach Reflections}

For the shock structure shown in Fig. 7, region 1 is supersonic. The centre portion of the Mach disk, BC, is a strong normal shock and region 2 is subsonic. Region 3 could be supersonic or subsonic, depending upon the strength of the separation shock. Both $\mathrm{BE}$ and $\mathrm{CF}$ being weak reflected shocks, region 4 is supersonic. Thus, flow downstream of the shock structure is a mix of subsonic flow at the nozzle core and supersonic flow near the nozzle walls. Region 3 and 4 comprise of separated flow.

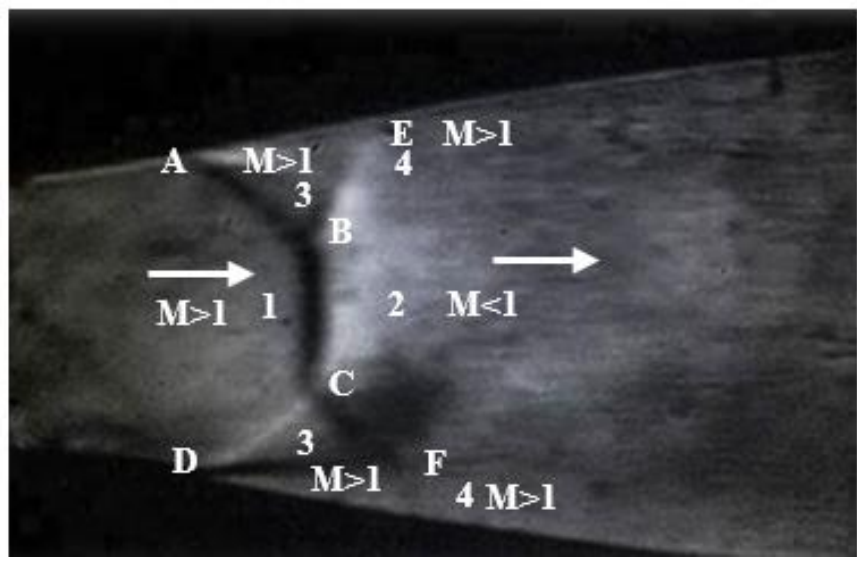

Fig. 7. Regions of flow

The shape of the Mach disk is determined by the nonuniformity associated with the flow upstream of the separation shock. The non-uniformities are governed by the presence of both axial pressure gradients $\left(\frac{\partial p}{\partial x}\right)$ and radial pressure gradients $\left(\frac{\partial P}{\partial r}\right)$ in the flow upstream. For supersonic nozzle flow, the axial gradient $\left(\frac{\partial p}{\partial x}\right)$ is $<0$, but $\left(\frac{\partial P}{\partial r}\right)$ can be positive or negative. For the shock structure shown in Fig. 8, the Mach disk BC, is convex in shape. This can be attributed to the type of pressure gradient $\left(\frac{\partial P}{\partial r}\right)<0$ present in region 1 . Such a pressure gradient increases from the nozzle wall towards nozzle axis, ie, pressure, $\mathrm{P}$ increases from A towards nozzle axis. The Mach number and shock strength also follow the same nature. Similar variation with respect to pressure gradient and Mach number can be observed in the subsonic flow in the region 2 .

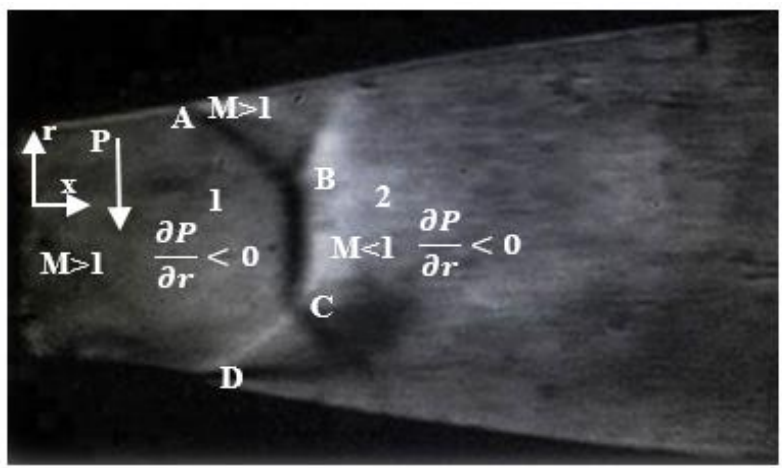

Fig. 8. Convex shaped Mach disk
The subsonic flow in the region 2, tries to achieve uniform pressure gradient by developing back pressure such that the strength of the shock decreases near the axis and increases whilst moving away from axis. This can be achieved by moving the shock downstream (to reduce shock strength near to axis) and upstream (to increase shock strength, moving away from axis) respectively. In doing so, the shock structure attains a convex shape at $\mathrm{BC}$ and the separation shock $\mathrm{AB}$ and $\mathrm{DC}$ become more oblique in nature. The presence of positive pressure gradient, $\left(\frac{\partial p}{\partial x}\right)>0$ implies an increase in pressure from axis towards wall and in this case the shock movement will be opposite and the Mach disk attains concave shape. This type of shock structure mostly happens in thrust optimised contoured rocket nozzles [3-5].

\section{After Shocks}

At NPR above 1.8, diamond shaped shocks, called aftershocks, were observed in the flow downstream.as shown in Fig. 8. The presence of aftershocks indicates that the flow downstream comprises of overexpansion and under expansion and the flow does not achieve equilibrium with a single compression shock. The dark pattern or fringes in Fig. 9 indicates regions of expansion i.e., under expansion in the flow downstream.

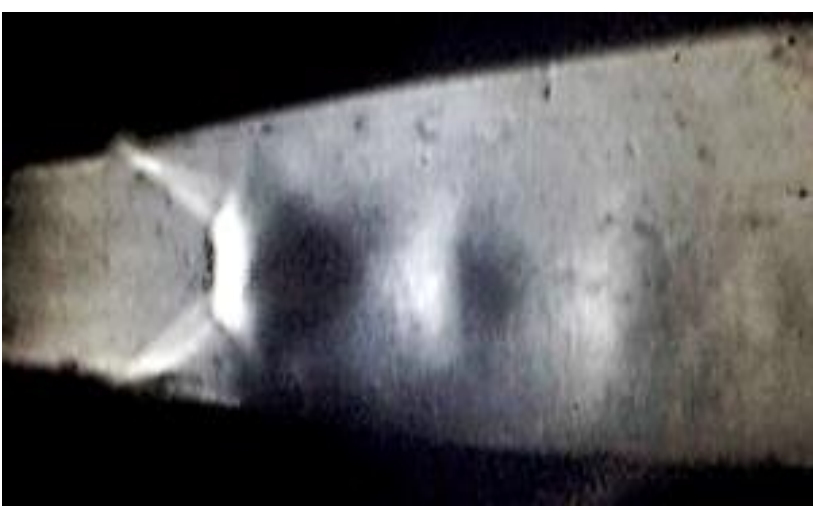

Fig. 9. Aftershocks

The formation of aftershocks can be better understood from the following schematic, Fig. 10 [6]. Even though the flow behind the Mach disk is subsonic, the wavy slipstream (with convergent-divergent shape) causes the subsonic flow to accelerate. The local sonic throats created by slipstream ensures supersonic flow downstream. The reflected shock after impinging on the shear layer reflects back as expansion fans, thus, causing the supersonic flow downstream to further accelerate. These expansion fans, whilst getting reflected from shear layer (jet boundary), reflect as weak oblique shocks and these appear as aftershocks. The aftershocks are predominantly straight thereby indicating that the flow downstream is more or less uniform in nature. 


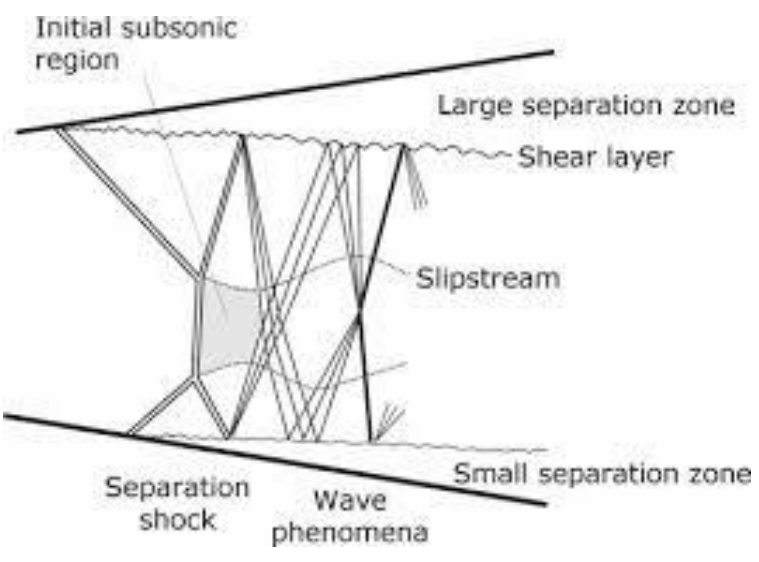

Fig. 10. Schematic of aftershocks [6]

\section{E. Shock Location}

Shock position $\left(\mathrm{A}_{\mathrm{s}} / \mathrm{A}_{\mathrm{t}}\right)$ is defined as the area ratio corresponding to the axial position of the Mach stem (normal part) of the shock and the throat area $\left(\mathrm{A}_{\mathrm{t}}\right)$. Image processing using MATLAB software was done to precisely predict the shock locations. The RGB images were converted to greyscale and a surface plot of pixels with respect to intensity was plotted. Fig.11 shows the comparison between processed and actual images.

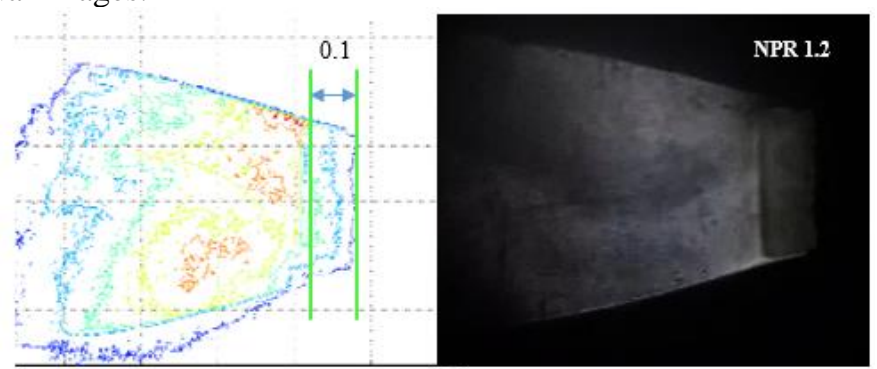

(a)
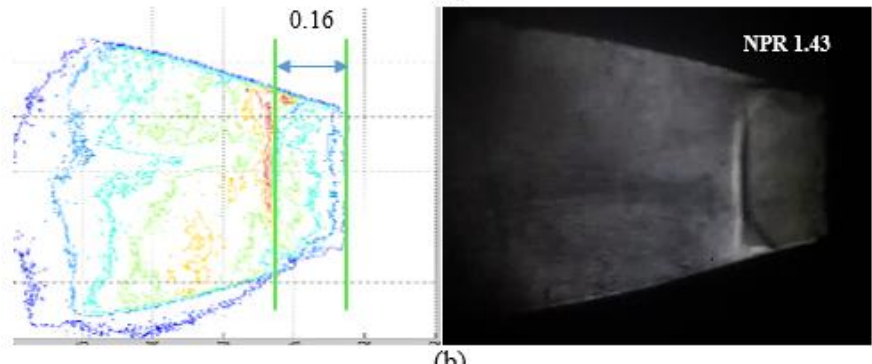

(b)
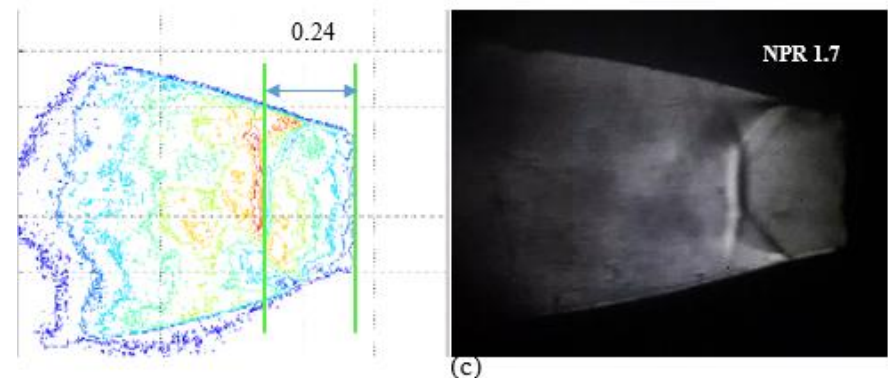

Fig. 11. Shock Locations

\section{F. Computational Analysis}

The computational domain was generated using Ansys and the Fluent module was utilized to solve the 2-D Reynolds Averaged Navier Stokes Equation (RANS) for the nozzle profile with the specified area ratio for various NPRs. Accurate mesh generation over the geometry to be analysed is a key step in the process of simulation as the quality of the mesh directly defines the accuracy of the solution obtained, the rate of convergence and the computational time required to run the simulation [10]. The mesh structure used in the nozzle profile is of the mapped face meshing using a triangle (best fit) pattern as it provides for a finer and denser packing of the grid within the limits of the geometrical boundaries. The generated mesh packs a denser fit within the throat section, which is region of primary interest. Details of the meshing parameters and the generated mesh on the nozzle profile are shown in Table 2 and Fig. 12 respectively.

TABLE 2. MESH DETAILS

\begin{tabular}{|c|l|c|}
\hline \multirow{2}{*}{$\begin{array}{c}\text { Ser } \\
\text { No. }\end{array}$} & \multicolumn{2}{|c|}{ Meshing Parameters } \\
\cline { 2 - 3 } & \multicolumn{1}{|c|}{ Parameter } & Value \\
\hline 1. & Relevance Center & Fine \\
\hline 2. & Initial Size Seed & Active Assembly \\
\hline 3. & Smoothing & High \\
\hline 4. & Span Angle Center & Fine \\
\hline 5. & Curvature Normal Angle & 18 \\
\hline 6. & Proximity Accuracy & 0.5 \\
\hline 7. & Minimum Size & $4.4153 \mathrm{e}-002 \mathrm{~mm}$ \\
\hline 8. & Maximum Face Size & $4.41530 \mathrm{~mm}$ \\
\hline 9. & Minimum Edge Length & $21.3310 \mathrm{~mm}$ \\
\hline 10. & Transition Ratio & 0.272 \\
\hline
\end{tabular}

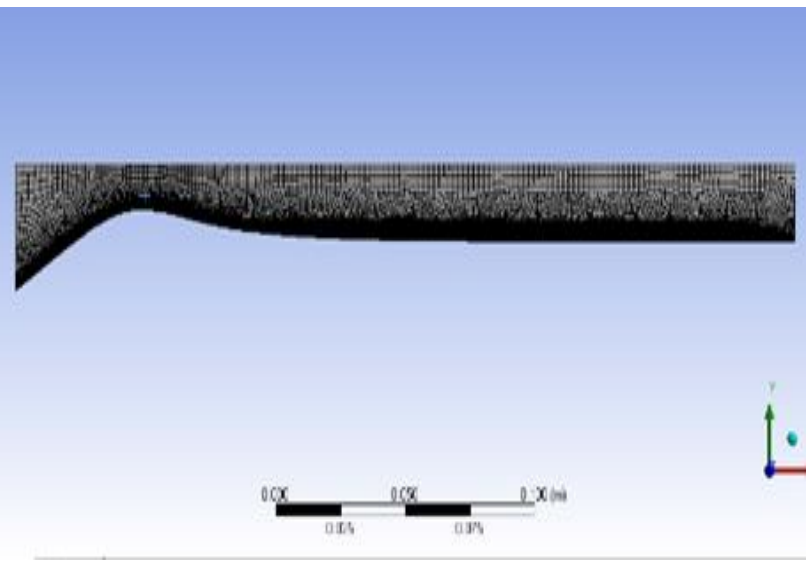

Fig. 12. Mesh generation

\section{G. Solution Setup}

The solution methods employed in the analysis followed an Implicit Formulation having a Least Squares Cell based gradient. First Order Upwind equations were used for Turbulent Kinetic Energy and Turbulent Dissipation Rate while Second Order Upwind was used for analysing the Flow. A hybrid initialization method was employed prior to running the calculation activities.

The solution setup involves defining the appropriate boundary conditions, flow properties and the stagnation flow properties. Since the flow dealt was in the supersonic regime, the solver used was density-based and axisymmetric across the 2D space to account for the entire geometry of the nozzle profile. To account for the prediction of shock location, 
2-equation, k-epsilon model was used in which the following constant values were assigned: -

$\mathrm{Cmu}=0.09 ; \mathrm{C} 1$-Epsilon $=1.44 ;$ C2-Epsilon $=1.92 ;$

TKE Prandtl Number $=1$, Energy Prandtl Number $=0.85$ and Wall Prandtl Number $=0.85$.

The boundary conditions at the inlet and outlet of the nozzle are defined in terms of pressure values. Reference values of temperature, pressure, density and ratio of specific heats were set at $288.16 \mathrm{~K}, 101325 \mathrm{~Pa}, 1.225 \mathrm{~kg} / \mathrm{m}^{3}$ and 1.4 respectively

\section{H. Simulation}

After setting the appropriate boundary conditions, the nozzle was tested for a range of inlet pressure values. In the course of locating the position of the shockwave, multiple flow parameters were recorded which included static and total properties along with the velocity variation along the nozzle.

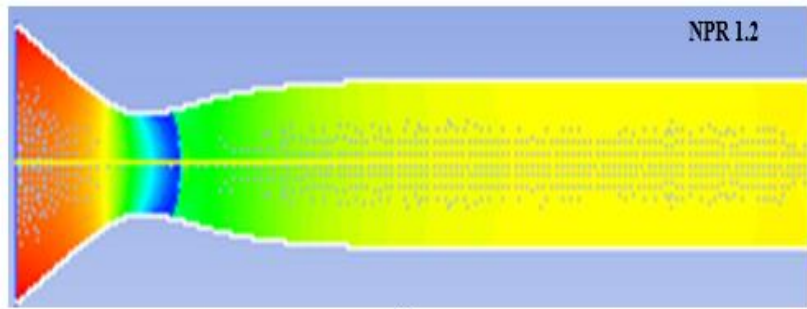

(a)

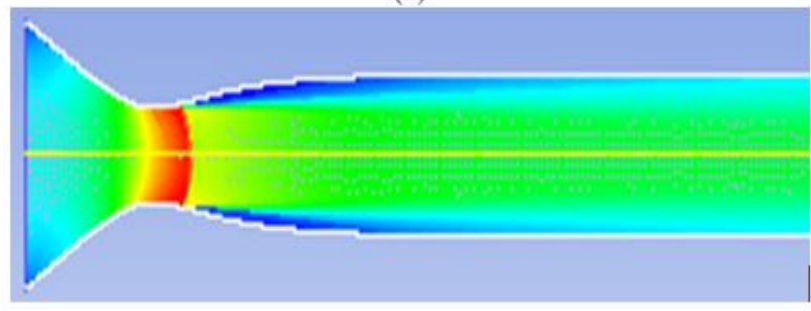

(b)

Fig. 13. (a) Velocity Contours (b) Static Pressure Contour

The contours of static pressure and velocity along the nozzle length for NPR 1.2 is shown in fig 13. The location of formation of normal shock is evident by the small region across which the static pressure increases drastically and a corresponding decrease in velocity as indicated in fig 13 (a) and (b) respectively and the corresponding shock location (x) is shown along the $\mathrm{X}$ axis. Similarly, the shock locations for NPR corresponding to 1.43 and 1.7 are shown in fig 14 (c).

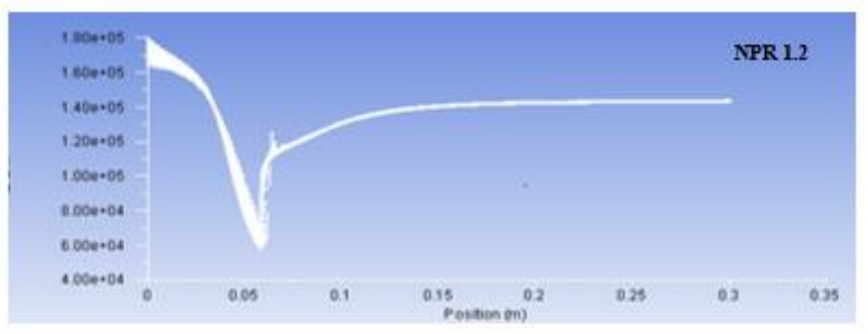

(a)

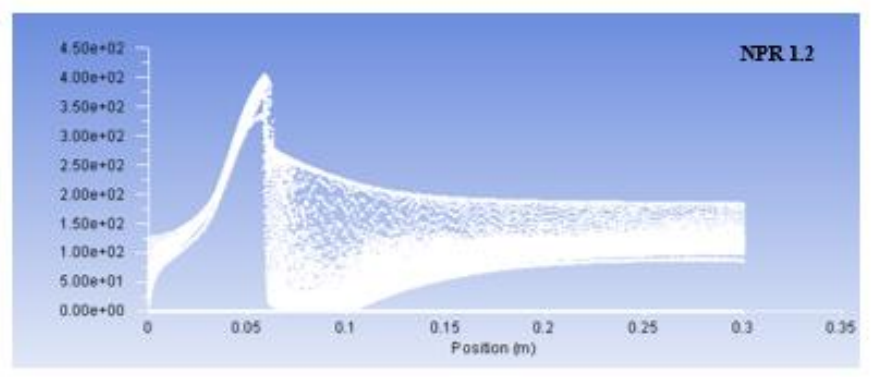

(b)

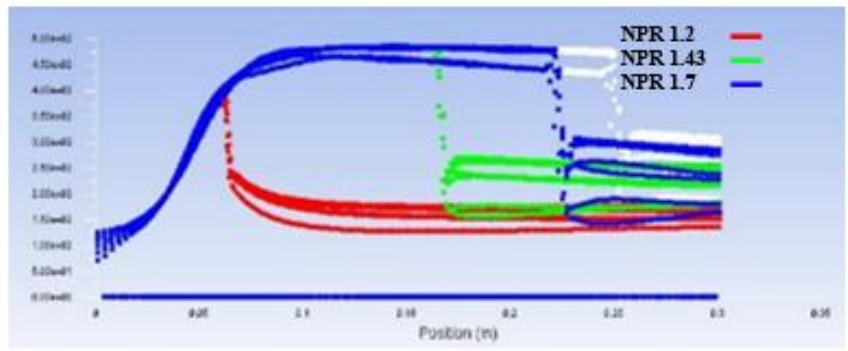

(c)

Fig. 14. Variation in flow properties across the shockwave. (a) Static Pressure for NPR=1.2; (b) Static Velocity for NPR=1.2; (c) Static Velocity comparison for NPR 1.2, 1.43 and 1.7

\section{Comparison of shock location}

The locations of shock (normal part of Mach stem) corresponding to NPRs $1.7,1.43$ and 1.2 obtained using experimental methods and computational simulation are shown as in table 3 .

TABLE 3 COMPARISON OF SHOCK LOCATIONS

\begin{tabular}{|c|c|c|c|}
\hline NPR & $\begin{array}{c}\text { Experimental } \\
\left(\mathrm{A}_{\mathrm{S}} / \mathrm{A}_{\mathrm{t}}\right)\end{array}$ & $\begin{array}{c}\text { CFD } \\
\left(\mathrm{A}_{\mathrm{S}} / \mathrm{A}_{\mathrm{t}}\right)\end{array}$ & $\begin{array}{c}\text { \% } \\
\text { Error }\end{array}$ \\
\hline 1.70 & 1.401 & 1.4873 & -6.1 \\
\hline 1.43 & 1.33 & 1.2759 & 4.13 \\
\hline 1.20 & 1.18 & 1.1511 & 2.449 \\
\hline
\end{tabular}

\section{CONCLUSION}

The fundamental investigation of shock formation of a contoured nozzle with area ratio 1.62 , defined by profile as shown in fig 1 has been undertaken. For $1.15 \leq \mathrm{NPR} \leq 1.3$, the flow field was dominated by weak normal shocks whose strength subsequently increased with increase in NPR. For $\mathrm{NPR} \geq 1.4$, the shock structure started developing lambda feet which was symmetric in nature. With the increase in NPR the feet of the lambda shock grew stronger and bigger asymmetrically. 
As the NPR was increased, the shock moved further downstream, causing SBLI induced boundary flow separation and a reduction in the Mach Stem. The shear layer emanating from the incipient/separation shock became more prominent, causing non-uniform detached flow with eddies downstream.

Beyond NPR $\geq 1.3$, the Mach stem developed a convex shape, due to the presence of negative pressure gradient $\left(\frac{\partial P}{\partial r}\right)<0$ in the non-uniform flow upstream of the shock as mentioned in fig 8 . The negative pressure gradient increases with increase in NPR and in the process of attaining flow equilibrium, the Mach stem becomes more and more convex, with the increase in NPR.

Subsonic flow downstream of the Mach stem expands to near-sonic speed and exhibits an alternation of subsonic and supersonic flow due to wavy slipstream. The expansion is due to the reflection of the trailing shock of the lambda foot from the separation pressure boundary. Eventually, the flow compresses to subsonic speed and matches with the ambient pressure.

A shock diamond occurs downstream of the shock suggesting the flow becomes subsonic, then accelerates and further becomes supersonic. This acceleration of local flow to supersonic flows is done by means of the slipstream acting as multiple sonic throats which gives the slipstream a repetitive convergent-divergent profile.
Comparative study of shock location suggests that there is not much difference between the actual shock position and the position predicted by standard k-epsilon model. The error increases with increasing NPR.

\section{REFERENCES}

[1] Verma S. B.: Study of Flow Separation in Truncated Ideal Contour Nozzle. Journal of Propulsion and Power, Vol.18, No. 5, SeptemberOctober 2002.

[2] Hunter C. A.: Experimental, Theoretical and Computational Investigation of Separated Nozzle Flows. AIAA-98-3107.

[3] Francesco Nasuti and Marcello Onofri, Shock Structure in Separated Nozzle Flows, Shack Waves (2009) 19:229-237, DOI 10.1007/s00193-008-0173-7.

[4] Abdellah, Hadjadj, Marcello, Onofri (2009).: Nozzle Flow Separation, Shock Waves (2009), 19:163-169.

[5] Stark R, Genin C.: Experimental Study on Rocket Nozzle Load Reductions. Journal of Propulsion and Power, Vol.28, No.2, MarchApril 2012.

[6] Papamoschou, Dimitri, and Andreas Zill. "Fundamental investigation of supersonic nozzle flow separation." In 42nd AIAA Aerospace Sciences Meeting and Exhibit, p. 1111. 2004.

[7] S. Mohapatra, P. N. Mohanty, H. S. Panda and T. K. Biswal, "Background Oriented Schlieren (BOS) Technique: An Effective Flow Visualization Tool for Intermediate Ballistics," 2019 International Conference on Range Technology (ICORT), Balasore, India, 2019, pp. 1-5, doi: 10.1109/ICORT46471.2019.9069668.

[8] Settles, Gary S., Elizabeth B. Hackett, James D. Miller, and Leonard M. Weinstein. "Full-scale schlieren flow visualization." Flow Visualization 7 (1995): 2-13.

[9] Mazumdar, Amrita. "Principles and techniques of schlieren imaging systems." (2013).

[10] Settles, Gary S., Elizabeth B. Hackett, James D. Miller, and Leonard M. Weinstein. "Full-scale schlieren flow visualization." Flow Visualization 7 (1995): 2-13. 